\title{
DIDÁCTICA DIGITAL
}

\section{Jeanne Yvanova Duarte Mora 1}

Universidad Pedagógica Experimental Libertador - UPEL

Instituto Pedagógico Rural Gervasio Rubio - IPRGR, Rubio, Táchira, Venezuela

http://dx.doi.org/10.5209/NOMA.56689

Resumen: Ante el avasallante despliegue que está desarrollando la tecnología en la comunicación, en los negocios, en las relaciones humanas, en el sistema financiero y hasta el proceso educativo; debido a la incorporación continua de equipos, programas y estrategias al servicio de una determinada función. Referido a ello, las organizaciones educativas, no han escapado del proceso de reestructuración e incorporación de herramientas tecnológicas, para mejorar sus servicios administrativos y masificar el desempeño de los docentes, que en este caso desempeñaran nuevos roles: de facilitadores, de tutores y de preparadores de material instrucciones. Ante, este reto, el sistema educativo en sí, requiere un cambio actitudinal y de compromiso de los involucrados, además de la inversión respectiva en infraestructura, en equipos, en paquetes y en la capacitación de los responsables de la actividad formativa.

Palabras clave: didáctica digital, organización, tecnología, usuarios, roles.

\section{Digital Teaching}

Abstract: Faced with the overwhelming deployment of technology in communication, business, human relations, the financial system and even the educational process; Due to the continuous incorporation of equipment, programs and strategies in the service of a certain function. Referred to, educational organizations have not escaped the process of restructuring and incorporation of technological tools, to improve their administrative services and to increase the performance of teachers, who in this case will play new roles: facilitators, tutors and preparers Of material instructions. In the face of this challenge, the educational system itself requires an attitude change and commitment of those involved, as well as the respective investment in infrastructure, equipment, packages and training of those responsible for the training activity.

Keywords: digital didactics, organization, technology, users, roles.

\footnotetext{
I Socióloga del Desarrollo UNELLEZ-Barinas. Magister de Innovaciones Educativas UPELIPRGR. Doctorante de Innovaciones Educativas UNEFA - Táchira. Doc. del Instituto Pedagógico Rural Gervasio Rubio - IPRGR. Miembro de Núcleo de Investigación: Núcleo de Investigación en Educación Rural - NIER. Jefe de Línea de Investigación: Desarrollo Comunitario Rural - DECOR. Jefe de Unidad de Extensión-USACE. Integrante del Programa Estímulo al Investigador - PEl en Venezuela.
} 


\section{Introducción}

La acción de transmitir, discernir y de comprender, se presenta en diversos escenarios en los cuales se reflejan actitudes, conocimientos y recursos que a través de una acción bilateral y reciproca de influye el saber de un discente y se mide el conocimiento de un facilitador. Que producto de esa necesidad económica-social de lograr unos determinados fines (moralizar-generar un determinado tipo de ciudadano-promover un tipo de trabajador), Se indago en diversos artes o estilos de transmisión, en los cuales ese legado se fomentó para organizar las actividades educativas; ello conlleva incentivar y orientar el aprendizaje.

Este proceso, no se realiza sin dirección ni finalidad; se reconoce que debe proyectarse con el requerimiento de un arte- de una ciencia- de una determinada práctica que a través de su carácter social se encargara de utilizar como son las instituciones y organizaciones educativas.

\section{Didáctica Digital}

La improvisación ha arropado todas las estructuras, esta deslave de hacer y ejecutar sin planear y dirigir se ha debido a la cotidianidad de las actividades y las acciones; en las cuales el conocer algo no induce que se maneje a precisión todos los aspectos y elementos requeridos en ese hecho. Para evitar este vicio muy frecuentado en todas las instituciones, acciones y tareas se debe asumir el compromiso de tomar una estructura de acción, una gestión de pensamiento y sobre todo una directriz de enseñar.

Ante ello se toma la posición de autores como Mello C., I. (1974), que en su planteamiento didáctico expresan "Mas dependerá de la improvisación; nos protegerá contra la superstición rutinaria, nos dará seguridad y eficiencia en nuestra labor, haciéndola más rica completa, continua e integrada ".(p.70). Este elemento, diseño o modelo que nos puede alejar de lo incierto y de sus posibles consecuencias, es lo que se tiende a denominar como didáctica. Consciente, de esta actividad, se desarrolla la interpretación y el papel que juega la didáctica. Pero hay que aclarar previamente ciertos conceptos: 
Parafraseando a Arredondo, Pérez y Aguirre (2008) Estos procedimientos, se enfocan en tres acciones:(a) planear,(b) realizar y (c) evaluar. Cada uno de ellos se presenta a continuación el Planear compete a analizar los programas del curso, estudiar las características de la población, ajustar los objetivos a las condiciones reales de la enseñanza, decidir los criterios de la evaluación y por ultimo considerar las diferentes medios didácticos. El realizar, induce a detectar la disposición, comunicar los objetivos y actividades, promover condiciones y motivaciones, organizar el material y establecer cada evaluación y como acción final La Evaluación, permite verificar los aprendizajes, detectar aciertos y debilidades y por consiguiente hacer las rectificaciones.

Algunos autores como, Fiore y Leymonie (2007) presentaron la Didáctica como, "La didáctica es un campo disciplinario especifico cuya tentativa es clarificar las situaciones de enseñanza y discutir los posibles epistemológicos, los posibles Psico-pedagógicos y los posibles pedagógicos "(p.1).Cuando se precisa como disciplina, involucra visión, misión, objetivos, metas de acción, recursos y evaluación respectivamente; pero no puede ser ajena a la forma de crear conocimiento a sus postulados psicológicos al servicio de la enseñanza y la práctica de los docentes en el aula.

Esta concepción, presenta los elementos estructurales que debe presentar la Didáctica en su estado practico, siempre dirigida al campo formativo, en el cual el posible epistemológico lo cubren las bases teóricas de la formación, los posibles psico-pedagógicos corresponde a las aptitudes y actitudes que deben propiciar esa formación y los posibles pedagógicos corresponden a las estrategias para llevar adelante la acción de enseñar. Los autores, presentan "... como el conjunto de actividades que organizadas por el enseñante, favorecen la adquisición de saberes culturales por parte del estudiante" (p.1). Este conjunto de actividades, recopilas las diversas estrategias instruccionales al servicio del educando, para permitir la respectiva maduración intelectual que se requiere en el aprendizaje.

Parafraseando a Fiore y Leymonie (2007), se podría indicar, que la didáctica no es normativa, ni tampoco prescriptiva; no consiste en una receta sagrada, ni se basa en dogmatismos ni en descubrimientos ancestrales; es producto de la práctica y por tanto no se puede generalizar, por depender de una temática respectiva, de un contexto, del docente y del alumnado. Otros postulados, como el de Litwin (1999) citado por Fiore y Leymonie (2007), expreso que la didáctica," entendemos a la didáctica como teoría acerca de las prácticas de la enseñanza significadas en los contextos socio-históricos en que se inscriben". (p.1). Esta teoría responde a las concepciones, temáticas, categorías y criterios de diferenciación propias de la actividad educativa. 
Expresa Martínez (citado por Cabero, 2007), la acción didáctica...

Supone una acción del diseño del proceso de comunicación a establecer, reflexión que se apoya en modelos metodológicos que han de favorecer el logro del fin que se propone. La metodología es la base del diseño y para que este sea posible se han de considerar numerosas facetas y contemplar, no solo las características de los sujetos, de los contenidos, de los objetivos, etc. Sino que también ha de adaptarse al sistema de transmisión de esos contenidos, a la disponibilidad o no de fuentes y recursos, a las posibilidades de interacción de los implicados en el proceso, básicamente profesores y alumnos, a las posibilidades o no de toma de decisiones sobre el proceso por parte del alumno, etc. (p.22).

Esta concepción de Martínez, ve a la práctica y acción didáctica como un proceso de investigación y experimentación; que debe considerar el contexto, los actores y las temáticas a plantear.

Pero, la didáctica no es solo un cuerpo coherente de métodos, requiere de un contexto y de unos involucrados. Al respecto, acude a una estructura social como son las instituciones educativas. Con respecto a la Educacion como sistema, presenta una estructura muy específica (Ministerios, direcciones, departamentos), con una función que es la de capacitar y formar al ciudadano de una determinada sociedad, y con unos determinados responsables que en este caso lo conforman toda la comunidad educativa. Pero adicionalmente, Freitas y otros (1999), le presentan:

La organización no es sólo un sistema social o técnico, requiere de estructuras e integración de las actividades humanas en torno a diversas tecnologías. El sistema técnico está determinado por los requerimientos de trabajo de la organización y toma forma de la especialización de aptitudes y conocimientos requeridos, los tipos de maquinarias y equipos utilizados, los requerimientos de procesamiento de datos y la disposición de las instalaciones. Cualquier cambio en el sistema técnico afecta a otros elementos de la organización. (p.4)

El autor, Martínez (citado por Cabero, 2007), expresa "los procesos de enseñanza son, en primer lugar procesos de comunicación y en segundo lugar intencionados. Se trata de acciones comunicativas cuya intencionalidad es hacer crecer el repertorio de conocimientos, actitudes y aptitudes de que dispone el receptor" (p. 21). Este crecimiento consiste en la acumulación de conceptos, teorías, criterios de diferenciación; en el cual el medio es la enseñanza y la intención la de informar e formar a un individuo o a un colectivo.

Que ha cambiado, como también lo ha hecho el mundo y sus productos. Pero, no con la misma velocidad ni con el mismo impacto. Esto se debe al escaso uso de la tecnología en sus prácticas. 
Al respecto, se debe comprender previamente que es este elemento. La Tecnología, es un concepto importante es el planteado por Kast y Rosenzweig (1988), el cual definen la tecnología como:

La organización y aplicación de conocimiento para el logro de fines prácticos. Incluye manifestaciones físicas como las máquinas y herramientas, pero también técnicas intelectuales y procesos utilizados para resolver problemas y obtener resultados deseados (p. 217).

Además, Kast y Rosenzweig (1988) señalan que la tecnología afecta la estructura de la organización, así como su subsistema psicosocial. Por ende, al introducir una nueva tecnología como la Tecnología de la Información a una determinada empresa, automáticamente afecta al resto de la estructura del sistema organizacional. Parafraseando a Kast y Rosenzweig, (1988), la introducción de la tecnología requiere de cambios en la actitud y el comportamiento de la gente implicada.

Continuando, con esta posición, se presenta la siguiente imagen en la cual se refleja las limitantes que presenta la tecnología en lo que se tiende a denominar la Innovación Educativa:

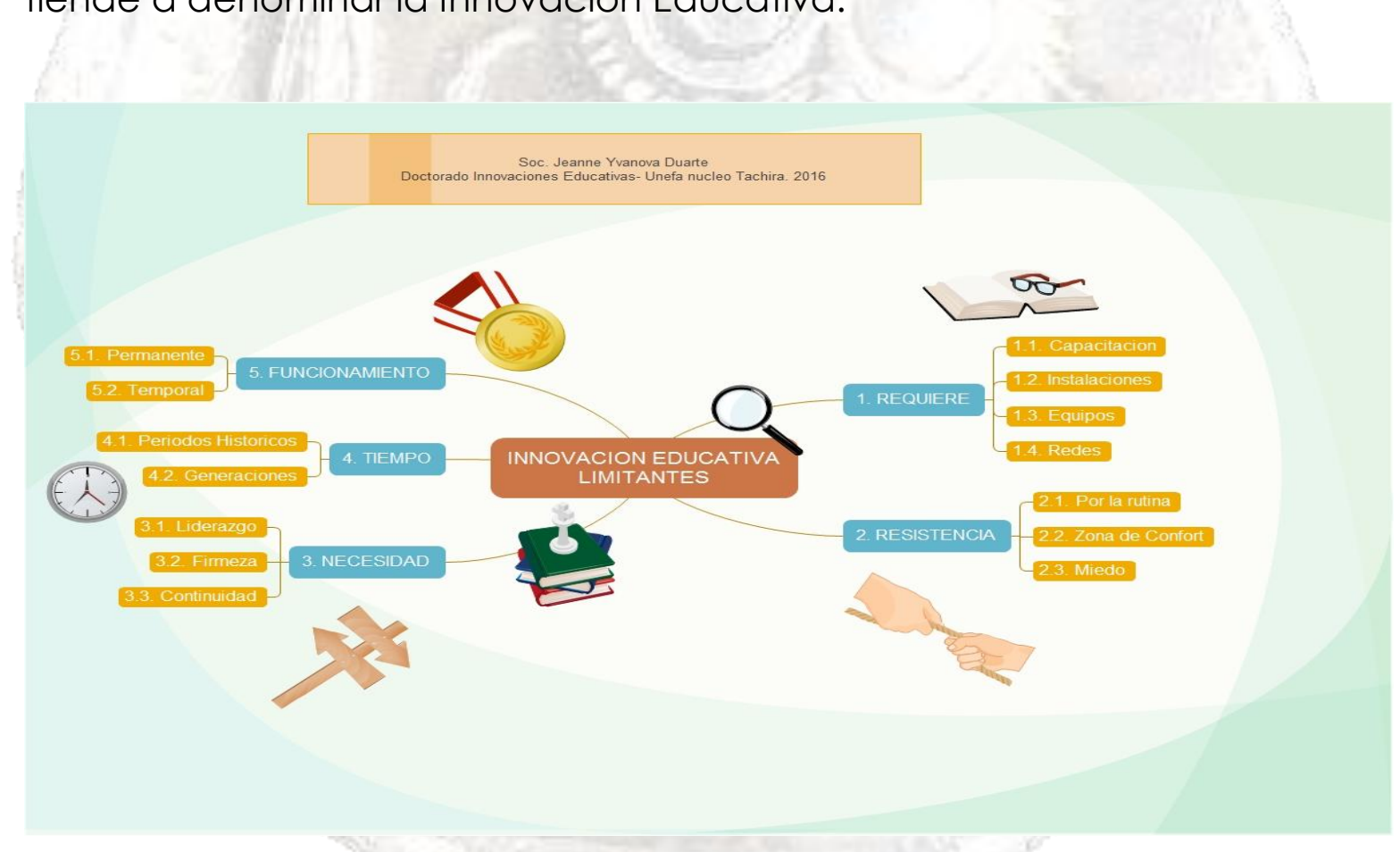

Fuente: Duarte, J. Y. (2016).Limitantes de la Innovación Educativa 
Las limitantes, llevan implícito los requerimientos, las resistencias, las necesidades, el tiempo y el funcionamiento para ser efectiva la aplicación de cualquier recurso de orden tecnológico en el aula y el acto educativo. Es decir, son un instrumento de mediación de aprendizaje. Parafraseando a Suarez (s/f), La educación es un proceso humano y humanizado cuya actualización está amparada en los adelantos tecnológicos, es decir, las nuevas tecnologías de información y de la comunicación son parte vital para un nuevo modelo pedagógico basados en nuevos instrumentos para el aprendizaje, que permiten el desarrollo de habilidades y saberes a través de los procesos telemáticos.

En otras palabras viene a satisfacer una visión pedagógica con uso de la tecnología en los procesos de aprendizaje, no solo de los procesos de formación de la persona, sino que tenga incidencia en los procesos mentales.

\section{Discusión de la Didáctica Digital.}

Como la didáctica o la estrategia respectiva, que utiliza las organizaciones educativas para formar y capacitar a sus educandos; es variada puede utilizar diversos medios para llegar a ello, como los Eblogs, la caza del tesoro, e-ponencias, e-conferencias, talleres virtuales, aulas virtuales, web quest, entre otros, permiten una conexión entre el educando y el educador, sin la presencia constante en un aula cerrada. Como Ugalde (2004), expreso en su artículo de Trasvase, "es un hecho irreversible, la actual globalización lleva una fuerte carga de inhumanidad y de empobrecimiento de la mayoría y es necesario y posible otro tipo de globalización..." (s/p), esta posición cae en el anacronismo económico, porque la globalización es única, homogénea y generalizada, no puede haber otra forma de globalización, solo puede sustituirse los elementos internos para generar otra forma evolutiva pero, nunca en si la misma.

Además, continuando con la posición del autor, este esquema solo puede ser sustituido con una transformación en el Sistema Educacion hacia el servicio de la humanidad, sustituyendo patrones de individualismo posesivo por solidario, de pobreza por trabajo y empleo, transculturización por identidad y nacionalismo, de poder unipolar por estados nacionales y de una reorganización de la economía en conformidad con las exigencias de la población y las necesidades educativas con parámetros modernas - tecnológicos. 
La automatización trae consigo la posibilidad de que todas las ideologías estrechas de la dirección y la división o distinción entre técnicos y trabajadores puedan ser superadas. Esta superación se debe a la educación, a los nuevos métodos de dirección, y sobre todo, a la tecnología. Pero la educación debe venir acompañada por un proceso administrativo que facilite la creación de estas estructuras. Los medios para lograr obtener una planificación educativa idónea deben incluir una evaluación de las necesidades y así mismo un análisis de los sistemas, para saber de esta manera en que contexto presente y futuro se encuentra la organización educativa.

Ante esta situación el docente debe adquirir un perfil de administrador, que incluye los procesos de aprendizaje, puesto que los estudiantes son el pilar fundamental de la institución, por lo tanto se debe conocer las necesidades y los posibles problemas para de esta manera poder implementar algunos procesos o procedimientos administrativos que conlleven a solventar las necesidades ya identificadas y mejorar los aspectos para la solución de problemas tanto intra como extra institucionales, en otras palabras, la educación debe ser también un proceso administrativo que permita determinar las capacidades, los conocimientos y las actitudes de los actores del hecho educativo para un máximo provecho del proceso de enseñanza y aprendizaje.

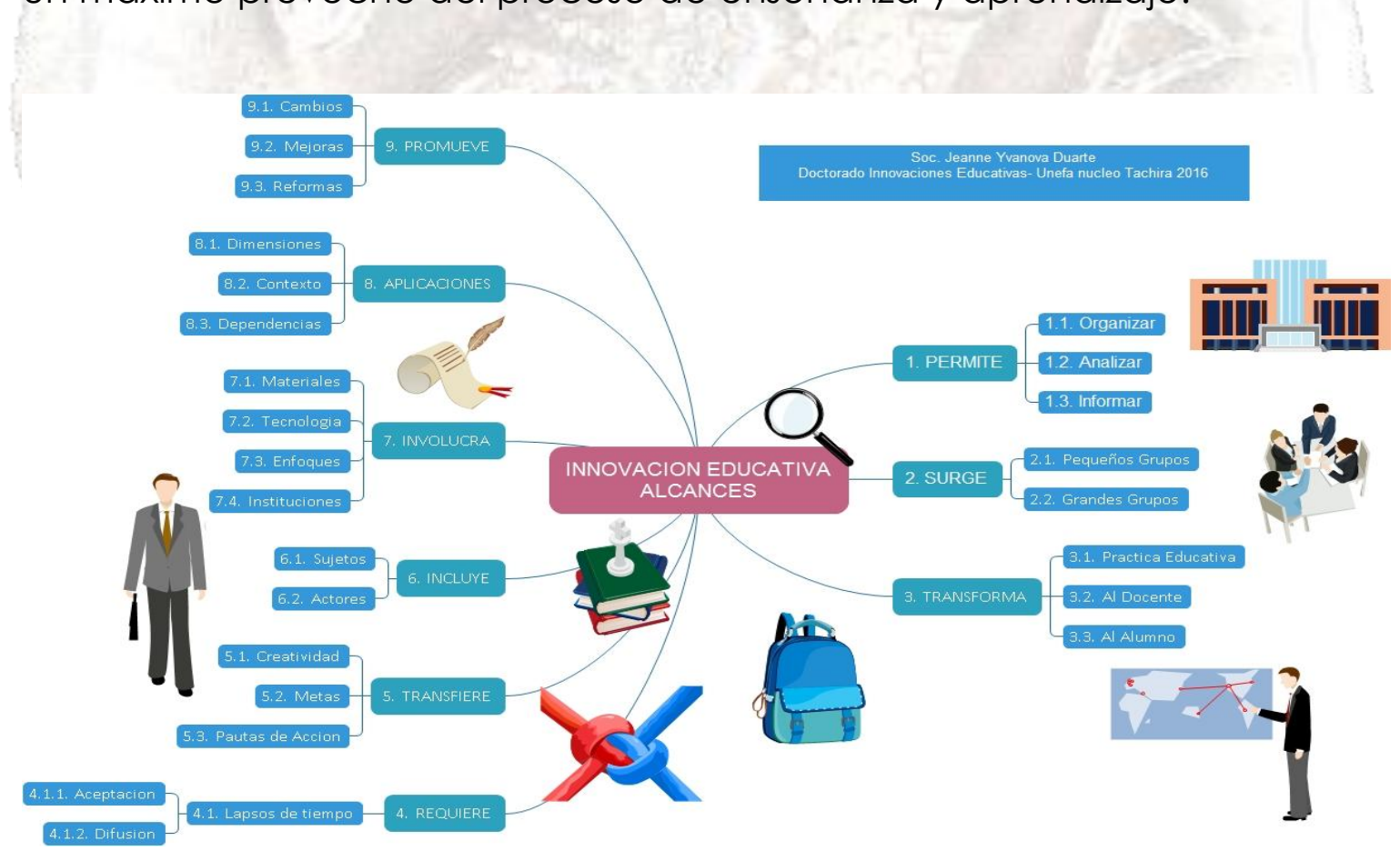

Fuente: Duarte, J. Y. (2016).Alcances de la Innovación Educativa 
Parafraseando a Lento, Neill y Gómez (2000, recopilado por Dede ) , los docentes tienen en su trayectoria laboral un poco estimulante relación con la tecnología, inicialmente con la radio y la televisión y luego con las computadoras $u$ ordenadores que es su nombre original; esto se debe al dogmatismo imperante con respecto al docente y el aula de clase y sobre todo al papel que debe jugar las tecnologías de la comunicación y de la información no como un elemento marginal en el aula , sino como una estrategia paralela de fundamentación.

Al respecto, los autores mencionados precisan "que toda utilización escolar de la tecnología refleja una determinada filosofía. Esta filosofía puede estar explicita en los planes y documentos de la escuela referentes a la tecnología, o puede estar implícita en el método general de enseñanza y aprendizaje de la comunidad escolar "(p.192).Esta concepción, refleja que debe tener una base teórica, epistemológica y hasta axiológica en cuanto a su implementación; no püede ser producto de la casualidad, ni del azar, ni de las circunstancias, debe tener una visión teórico-operativa para lograr un determinado producto en el docente y por supuesto en el alumnado.

Pero, no solo requiere de esta proyección, se debe permitir un enfoque más constructivista, para continuar como Lento, Neill y Gómez (2000, recopilado por Dede) precisa "... producir materiales menos definidos, que dejan a los docentes en libertad de elaborar sus propias versiones" (p.193). Y esta versión debe estar bajo criterios referidos: a) edad del alumnado, b) contexto educativo, c) habilidad del docente, d) formación tecnológica del docente, e) contenidos a presentar, f) creatividad y otras habilidades respectivas .

¿Pero, estos no son los únicos respaldos necesarios para incorporar la tecnología en el ámbito escolar y educativo?; requieren de equipamiento e infraestructura adecuada, de reparaciones y mantenimiento continuo. "internet es un lugar cambiante y dinámico, y el aula conectada a la red requiere una serie de nuevas expectativas por parte de docente y alumnos." (p. 198); esta expectativas son heterogéneas y cambiantes depende de la madurez de los actores y del momento histórico social en el cual se esté presentando.

Pero, ello no es solo lo único requerido, para no malgastar en inversión, debe tener personal técnico incorporado en el recinto educativo e implementar cursos permanentes de capacitación para los docentes además de ofrecerles incentivos por dicha formación. 
Como John Dewey, expreso "la educacion no es una preparación para la vida; es la vida misma" ; esta sencilla expresión tiene un marcado peso sincrónico, es la educacion es un proceso continuo y necesario para sobrevivir, para coexistir, para compartir y para vivir; en lo cual las nuevas funciones, responsabilidades y oportunidades desvían la educacion a un proceso social interactivo, en el cual la tecnología desempeña su papel de herramienta que permitirá el alcance de nuestras ideas y participación. Este proceso social interactivo a su vez permite que se construyan ideas, se valoren, se apliquen, las preserven 0 extingan y hasta las permitan compartir, por medio de la tecnología y toda su comunidad compleja: narradores, editores, artistas, fotógrafos, educadores, alumnos, programadores, entre otros.

Las denominadas "islas de innovación", pasaran a archipiếlagos y luego a continentes, si las barreras del costo de la tecnología, su rápida evolución y los conocimientos especiales se van minimizando gradualmente; esta minimización puede generar un efecto inverso, con más docentes inmersos en el uso de la tecnología como herramienta de trábajo, con más inversión en equipos para uso escolar, con continua capacitación de la planta docente evolucionara el sistema educativo. Para ello se debe, eliminar de la conciencia colectiva el miedo hacia las maquinas, de su rivalidad profesional y de comprender que: automatizar no significa deshumanizar.

\section{Referencias}

Arredondo, Pérez y Aguirre (2008). Didáctica general (manual introductorio). 3era edición. México: Limusa.

Cebero, J. (2007) Cop. Nuevas Tecnologías Aplicadas a la Educacion. Ed. Mc Graw Hill.1era. Edición. Madrid.

Dede, Ch. (2000). Aprendiendo con tecnología. Comp. Colección Redes en Educacion. Argentina: Paidos

De Freitas, V. y Viloria, O. (1999). Factores tecnológicos que inciden en la adopción de las herramientas CASE en las organizaciones Venezolanas. [Documento en línea] Disponible en: http://www.revistaespacios.com/a99v20n02/30992002.html [Consulta: 2016, Noviembre 25]

Fiore F., E. y Leymonie S., J. (2007). Didáctica Práctica. (Para enseñanza media y superior). Ed. Grupo Magro .1 lera. Edición. Uruguay: Montevideo Landeau, R. (2012). Metodología y Nuevas Tecnologías. Colección Tropicos/Educacion. Caracas, Venezuela: Edit. Alfa. 
Hopper, M.D. (1990); Rattling SABRE New Ways to Compete on Information. Harvard Business Review. May-June.

Kast, F. y Rosenzweig, J. (1988); Administración en las Organizaciones. Enfoque de Sistemas y de Contingencias. México: McGrawHill/Interamericana de México, S.A. de C.V.

Mello C., I. (1974). El Proceso Didáctico. Argentina: Edit. Kapeluz S.A.

Suarez, C. (s/f). Los entornos virtuales de aprendizaje como instrumento de mediación. [Artículo en línea] Disponible en: http://www.quadernsdigitals.net/index.php?accionMenu=hemeroteca. VisualizaArticulolU.visualiza\&articulo_id=7548 [Consulta: 2016, Noviembre 25]

Ugalce, L. (2004), Universidad, valores, espiritualidad y globalización. Revista Electrónica Educere Trasvase: oct- nov.-dic. Año 8 № 27. Mérida, Venezuela. 